\title{
Bearing Time-to-Failure Estimation using Spectral Analysis Features
}

\author{
Reuben Lim Chi Keong ${ }^{1,2}$, David Mba ${ }^{1}$ \\ ${ }^{1}$ Cranfield University \\ ${ }^{2}$ Republic of Singapore Air Force \\ r.limchikeong@cranfield.ac.uk
}

\begin{abstract}
With the increasing use of health usage monitoring systems on helicopters, a lot of research has been under taken for diagnostic of transmission components. However, most of these works are performed in laboratory environments and there are hardly any published works on in-service application. In this study, we present an experience in diagnosis of a helicopter gearbox bearing using actual service data gathered from AH64D helicopters belonging to the Republic of Singapore Air Force. A number of helicopters have been found with grease leak and radial play in the Tail Rotor Gearbox (TRGB) output shaft during field maintenance. Subsequent tear-down inspections of the TRGBs revealed that they had similar defects of bearing races spalling and widespread pitting of the rolling elements. Spectral analysis was carried out on the accelerometer data from these helicopters and correlated with the teardown inspection findings. The fault patterns exhibited corresponds well to progressing stages of bearing wear and are consistent across defective gearboxes from different helicopters. It is demonstrated that simple spectral analysis can be effective in tracking progressive stages of bearing damage using both low and high frequency bandwidths. The observed fault patterns are extracted as features for diagnosis and used to determine the bearings estimated time-tofailure for maintenance planning.
\end{abstract}

\section{Introduction}

Since the early 1990s, the use of Helicopter Health Usage Monitoring Systems (HUMS) has been increasingly prevalent due to the potential benefit of enhancing flight safety and reducing maintenance costs. In most applications, HUMS uses embedded accelerometers to monitor the health of mechanical components. When damage initiates and progresses in components, they can be diagnosed through fault patterns in the vibration signatures. Features from these signatures can then be extracted as a measure of component health and used to predict the remaining safe operating life of the component. These features provide warning of potential failure and allow maintenance to be performed as required as opposed to traditional scheduled maintenance. There are several vibration based methods to date to diagnose bearing faults and they can be broadly classified into (1) time domain, (2) frequency domain and (3) time-frequency methods as discussed in [1;2]. Time domain methods use the descriptive statistics such as mean and kurtosis of the time-series signals itself to identify bearing faults. Autoregressive modeling of the vibration time series is another popular time domain method and it has been applied together with neural networks for bearing fault detection [3]. For frequency domain methods, the use of the Fast Fourier Transform (FFT) of the vibration signal is widely used to identify fault frequencies in rotating machineries. The direct use of descriptive statistics of the fault frequencies within the FFT spectra has been 
shown to be a simple but effective method to diagnose faulty bearings in [4;5]. Bearing fault frequencies, however, are often masked by more dominant gear mesh frequencies and envelope analysis is a popular technique used to identify them by demodulating the high frequencies impulses caused by the bearing faults [6]. Time-frequency methods are relatively recent where the vibration signal is no longer assumed to be stationary and techniques using wavelet analysis and Wigner-Ville distribution are applied to detect changes in the frequency content of the signal [6]. As mentioned by Sikorska et al [7], most of the research works using these methods are performed in a laboratory environment with seeded fault testing and there are little published works on complex components exposed to normal operating environments. This study focuses on the actual service experience gathered from HUMS equipped AH64D helicopter belonging to the Republic of Singapore Air Force (RSAF). Vibration-based condition monitoring data from different helicopters with in-service defects found on their Tail Rotor Gearbox (TRGB) are correlated with tear down inspection findings.

\section{AH64D TRGB \& HUMS Description}

The analysis discussed in this paper focuses on the TRGB output shaft thrust bearing in the AH64D helicopter. The TRGB is grease lubricated single stage gearbox and serves to transmit drive torque from the intermediate gearbox to the tail rotor system. An assembly drawing of the gearbox and the location of the accelerometers used to monitor this component are shown in Figure 1.

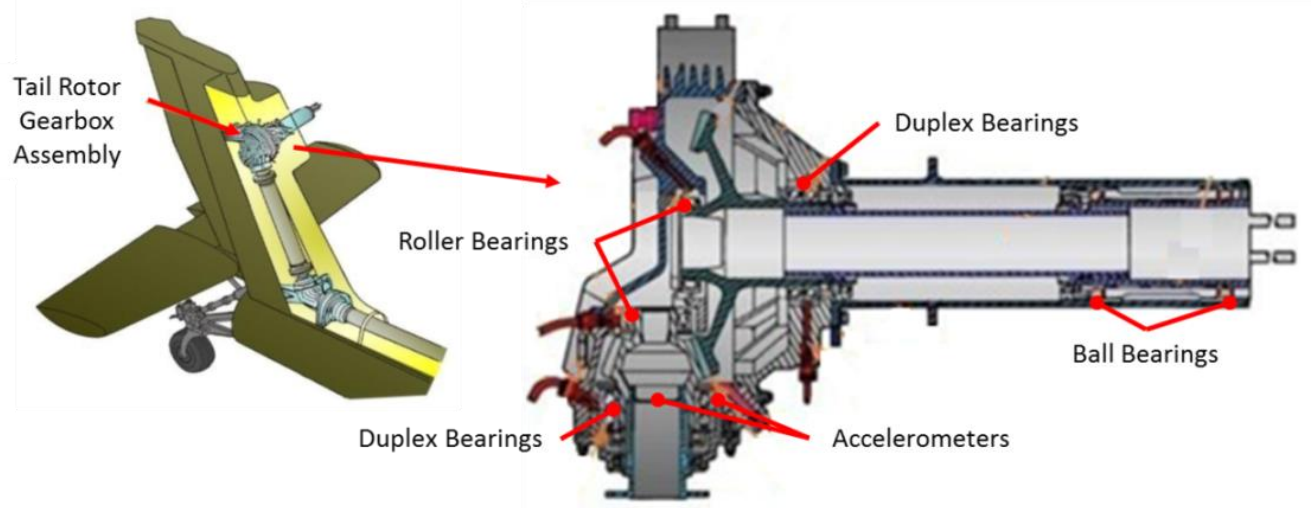

Figure 1 AH-64D tail rotor gearbox location \& assembly layout (Adapted from [4])

For the TRGB, there are two accelerometers measuring both vertical and lateral directions. The HUMS data on the RSAF AH64D helicopter is acquired using the IAC-HUMS from Honeywell. The on-board systems measures vibration levels whenever the aircraft is on ground with rotors at flat pitch and rotating at 101\% RPM. This provides a controlled flight condition in which the vibration measurements are taken. The accelerometer measurement on the TRGB is acquired at a sampling rate of $48 \mathrm{kHz}$ and filtered using a Hanning window to reduce spectral leakage. Several sets of measurements are further asynchronously averaged with no overlapping applied to reduce sporadic noise in the signal. Due to limited onboard data storage capacity, the time domain data are not stored for post flight downloads and only the FFT spectrums are available for further processing and analysis. 


\section{Gearbox Bearing Defect Description}

From maintenance records, three TRGB was found with grease leaking from the output seals and upon further inspection, the output shaft was found with excessive radial play. These TRGBs for helicopter 1, 2, 3 had accumulated 1204, 1171 and 962 flying hours respectively. They were then removed for disassembly and further teardown inspection. After the disassembly, the ball bearings inside the outboard shaft were found to have extensive pitting on ball elements and spalling in the bearing races as shown in Figure 2a.
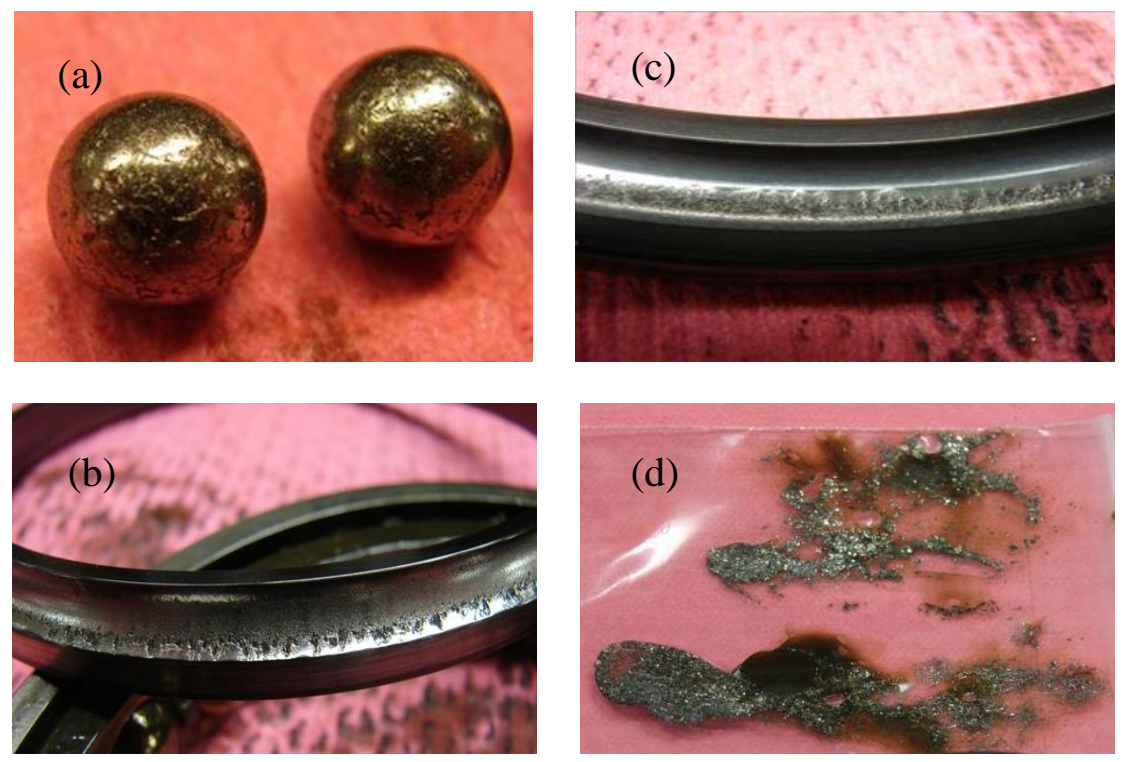

Figure 2 (a) Pitting on ball bearing elements, (b) Spalling on Inner race, (c) Spalling on Outer race, (d) Wear debris from removed grease sample

The running path of the wear pattern in the outer race is axially displaced (Figure $2 b$ ) and the pattern in the inner race is the widest in the radial load direction (Figure 2c). This is characteristic of bearing wear under both axial and radial loads as guided in [8]. There was significant amount of wear debris found in the grease (Figure 2d) and it was evident that the radial play of the shaft was caused by the deteriorated bearing. Evidence of heat oxidation was found on the quill shaft as well. It is likely that the damage in the bearing is caused by corrosion which initiated from moisture intrusion through leaking output seals; a common defect reported for the AH64D TRGB which was also reported in [9]. When grease leaks are found, the leak could not be repaired in the field and an unscheduled replacement of the TRGB has to be carried out. Such unscheduled replacement causes aircraft unavailability and significant man-effort for recovery. As such, it is desired for such defects to be detected earlier and for the replacement to be performed during scheduled maintenance.

\section{Bearing Fault Progression}

The fault patterns exhibited by progressive stages of bearing damage are well established in industrial applications as described in $[10 ; 11]$ and shown in Figure 3. 

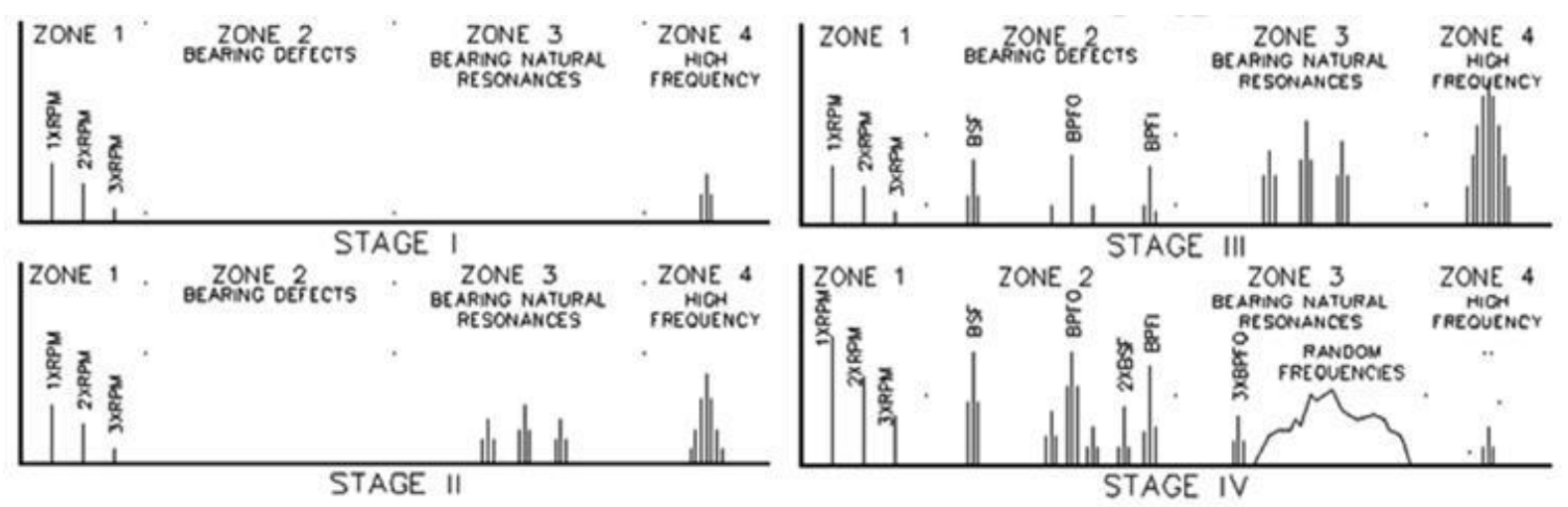

Figure 3 Fault patterns of bearing damage stages [10]

In Stage I, micro-defects and crack initiation causes ultra-high frequency activities. These activities are typically monitored using Acoustic Emission; such as in [12], rather than accelerometers. In Stage II, the micro faults develops into pits which begins to excite bearing elements and causes signals associated with their natural frequencies to be appear. Enveloping analysis is commonly used to demodulate a selected high frequency bandwidth of the FFT spectra and extract the bearing defect frequencies in this stage. As the pits become larger, fundamental bearing defect frequencies and their harmonics can be observed from the FFT spectra. Depending on the extent of the damage, these frequencies can be modulated by the shaft frequency and be observed as sidebands. Stage IV is the final condition before bearing catastrophic failure. As the defect becomes widespread, the bearing elements vibrate more randomly with the higher clearances. The localized defects may also have 'smoothen' out which reduces the signature of the periodic vibration as described in [13]. As such, the distinct bearing defect frequencies diminishes as an increase in noise floor or 'haystack' rises in the higher frequencies ranges.

\section{AH64D TRGB Spectral Analysis}

The available measurements from the three helicopters prior to and after replacement of the defective TRGB were obtained for analysis and compared with the bearing damage model described above. For two of the helicopters, gaps in the data history exist but it does not affect the study significantly as the trends from the FFT spectrum plots can still be clearly observed. HUMS data from another helicopter with a serviceable TRGB and with similar operating hours were also obtained for comparison with the defective gearbox. In this study, the HUMS data from the lateral accelerometer are used as its vibration signature showed a clearer response compared to the other two accelerometers. The reason for this is not investigated here though it is likely that there is less noise in the lateral direction in the environment. In their work using the AH64D Tail Rotor Test Rig, Goodman et al [4] has also observed that the lateral accelerometer is more sensitive to conditions within the TRGB. The bearing defect frequencies for the TRGB bearing are shown in Table 1.

Table 1 Angular Contact Bearing Defect Frequencies

$\begin{array}{ll}\text { Ball Pass Frequency Inner race, BPFI (Hz) } & 294 \\ \text { Ball Pass Frequency Outer race, BPFO (Hz) } & 244 \\ \text { Ball Pass Frequency, BF (Hz) } & 107\end{array}$


Figure 4a shows the Time-Frequency plot of the acceleration FFT spectrums against flying hours for a serviceable TRGB. Figure $4 \mathrm{~b}$ shows a magnified view at a lower frequency range that shows the evident vibration signatures. These include the Tail Rotor Shaft Frequency and the Gear Mesh Frequency (GMF) of the TRGB and the Intermediate Gearbox (IGB) frequency, together with their harmonics. Sidebands modulated at the Tail Rotor Pylon Shaft Frequency can also be observed surrounding the TRGB and IGB GMF. Figure 4c shows a snapshot of spectral plot at $\mathrm{t}=800 \mathrm{FH}$ and the mentioned frequency contents. From Figure 4, the magnitude of the spectral peaks at the GMF and their sidebands is stationary and does not show any trends over time for a serviceable TRGB.

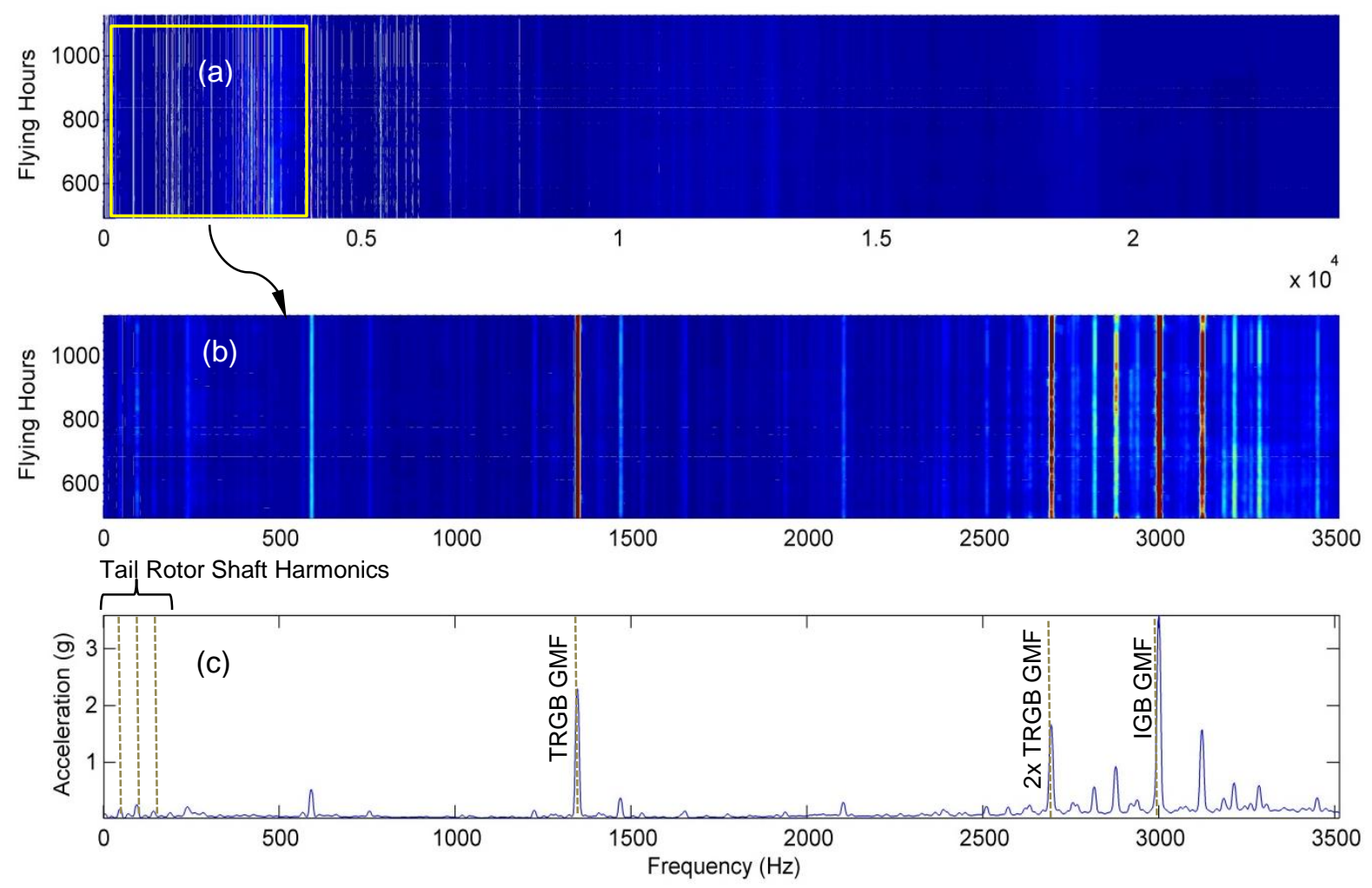

Figure 4 Time-Frequency plot of acceleration for a serviceable TRGB 

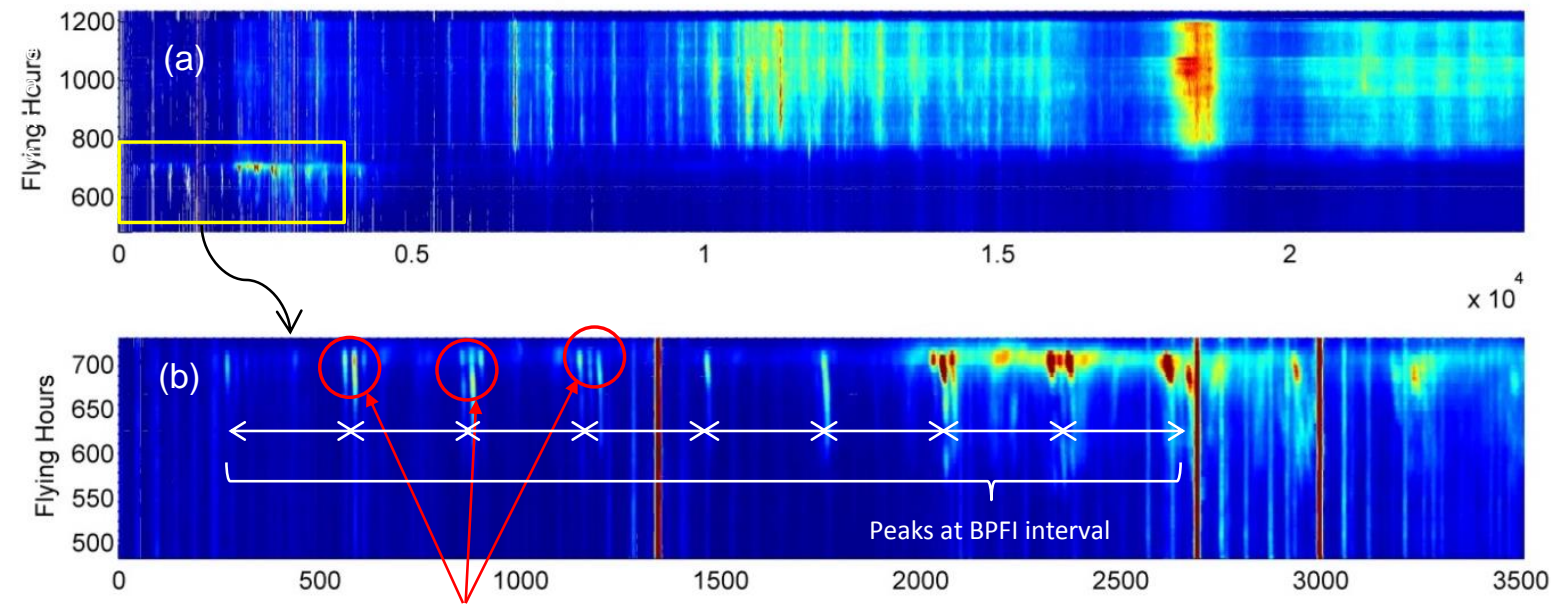

Harmonic peaks with sidebands

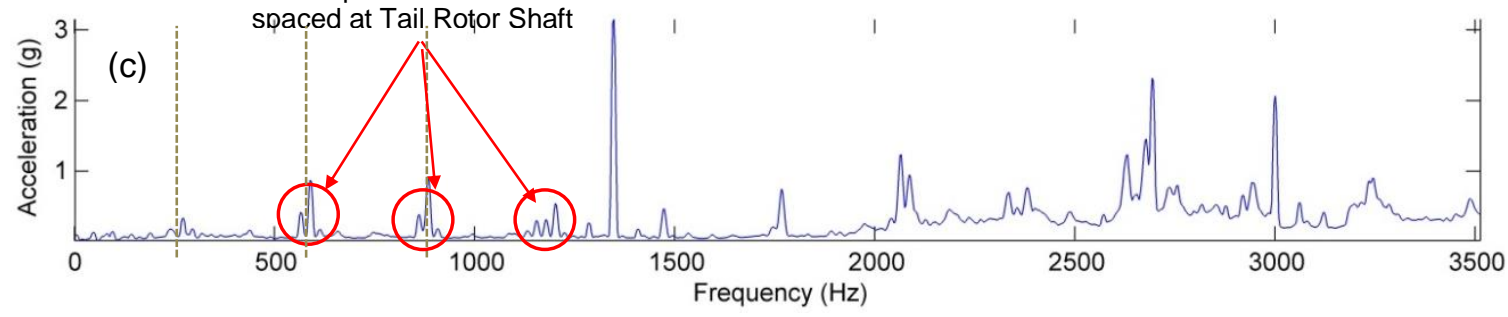

Figure 5 Time-Frequency plot of defective TRGB \#1 acceleration FFT
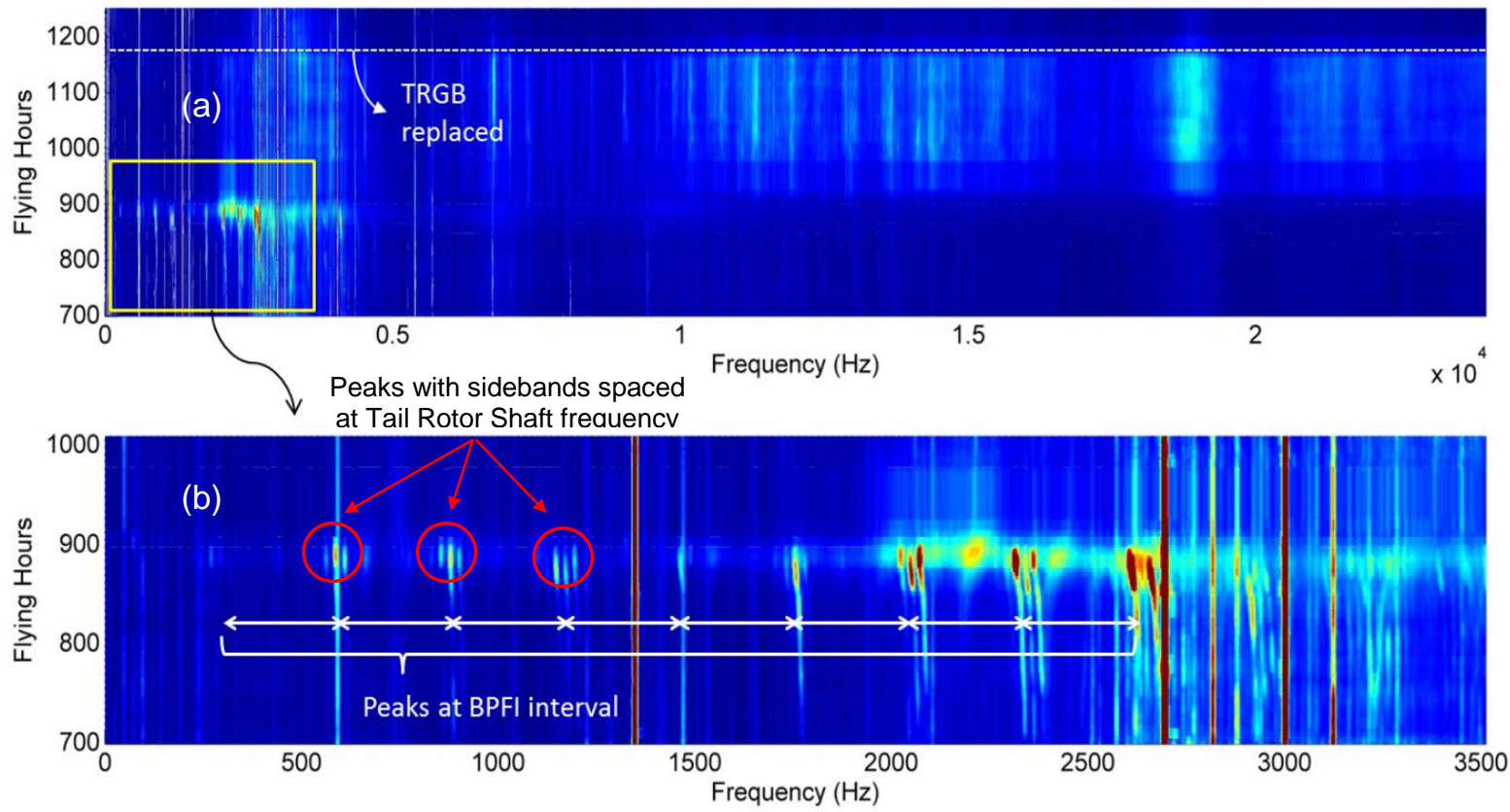

Figure 6 Time-Frequency plot of defective TRGB \#2 acceleration FFT 

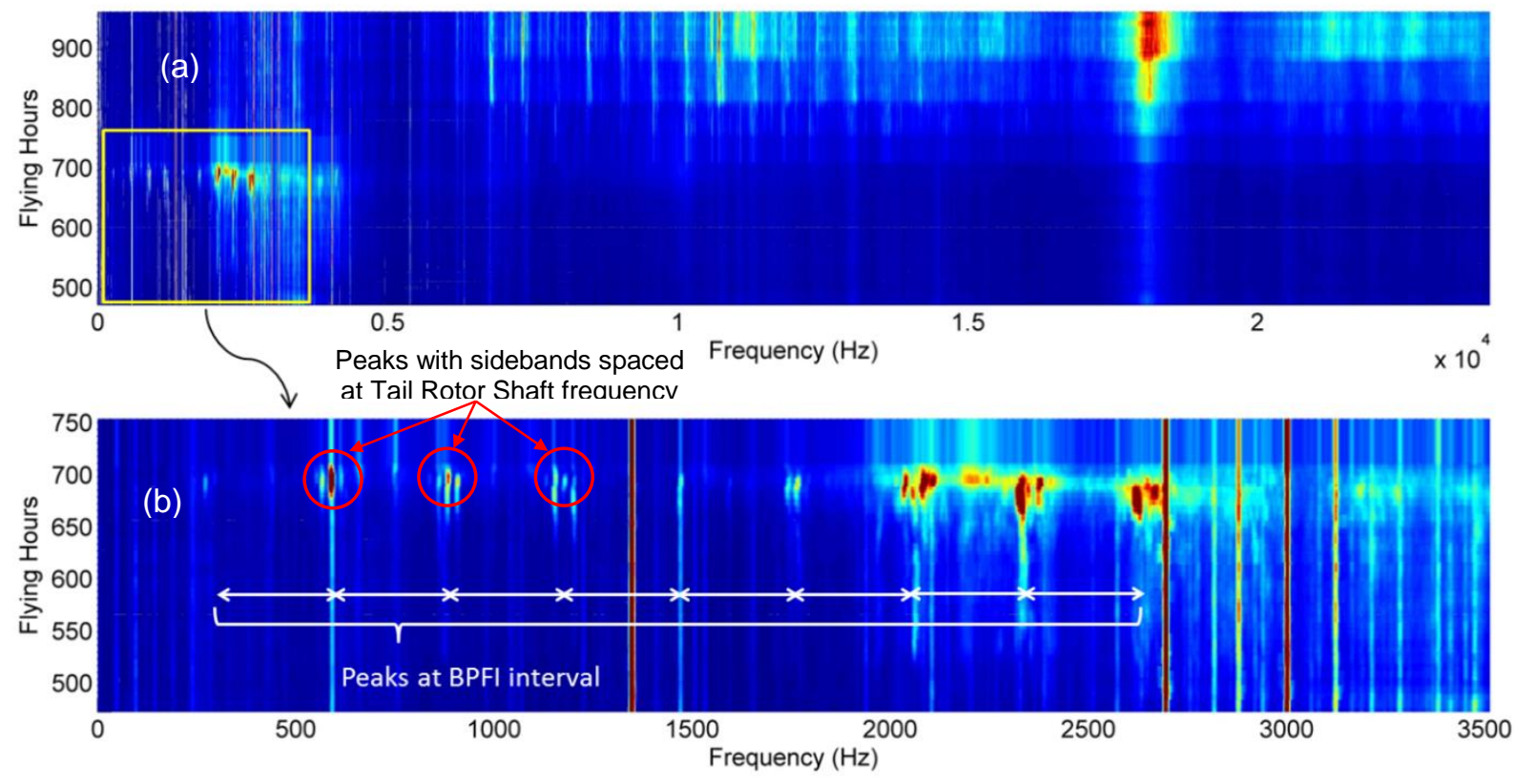

Figure 7 Time-Frequency plot of defective TRGB \#3 acceleration FFT

For comparison, Figure 5 shows the similar Time-Frequency plots of the FFT spectrum against time for the defective TRGB \#1. The dominant gear mesh and sideband signatures are still present but several fault patterns that differ from the serviceable TRGB plot are apparent. A key observation is that there are distinct changes in fault patterns at different frequency bands. From Figure 5a, it can be seen that there is a steady increase in spectral peaks within the $0-5 \mathrm{kHz}$ band. These peaks are spaced at the output shaft BPFI and accompanied by sidebands modulated at $1 \mathrm{x}$ tail rotor shaft frequency as shown in Figure 5b and Figure 5c. From [11], this is likely due to the bearing defect frequency acting as carrier frequencies for the shaft speed frequency. The presence of multiple harmonics of these peaks strongly suggests defects in the bearing inner race. These fault patterns agrees very well with Stage III bearing damage described above. This is further ratified from the severe spalling pits found in the inner race as seen in Figure $2 \mathrm{c}$ above. The vibration energy at this lower frequency band then diminishes and is followed by increase in spectral peaks at higher frequency band above $10 \mathrm{kHz}$. This agrees very well with Stage IV bearing damage and can again be supported by Figure 2c, where it can be seen that the damage is widespread in the bearing races and the rolling elements. These fault patterns in the FFT spectrum are also consistently observed in TRGB \#2 and \#3, which shared the similar bearing defect findings as shown in Figure 6 and Figure 7. When the TRGBs are replaced, the fault patterns are no longer present and the spectrum reverts to that of a serviceable TRGB with the associated gear mesh frequencies as seen in Figure 4. From the spectral plots in Figure 5 to Figure 7, the degradation of the TRGB output shaft bearings in the field environment are shown. All three TRGB have the same reported defects of grease leak and free play in bearings and the repeatability in the fault pattern demonstrates that simple spectral analysis can be effective in tracking progressive stages of bearing damage of actual helicopters in the field environment. 


\section{Feature Extraction}

From the spectrum plots, features are extracted from statistics of the fault patterns at different frequency bands to diagnose the health state of the bearing. From [4; 5], Bearing Energy (BE) features were developed using the Root-Mean-Square (RMS) energy of the frequency magnitude and was shown to be effective in detecting various bearing faults in seeded tests. As the fault patterns can be easily observed in the spectrum plots, the use of bearing energy features is adopted here. As there are both Stage III and IV damage stages, BE feature is developed for each stage. For Stage III, the RMS energy of the frequency magnitudes in the low frequency band of $250-2500 \mathrm{~Hz}$ is used to capture the spectral peaks of the BPFI harmonics. A rejection band of 0 to $250 \mathrm{~Hz}$ and $1250-1600 \mathrm{~Hz}$ are applied to eliminate effects from the tail shaft frequency, the dominant gear mesh frequencies and their sidebands. This feature monitors the extent of localised spalling within the bearing in Stage III. For Stage IV, the RMS energy in the high frequency band of $10 \mathrm{kHz}$ to $24 \mathrm{kHz}$ is adopted. It should be noted that this high frequency band is often the demodulation band used in envelope analysis to detect incipient defects. It is used here however as a measure of widespread damage in the bearing. The low and high frequency bands feature trends plots for the three TRGBs are shown in Figure 8. Although there are gaps in the data, the overall trends of the features are still obvious.
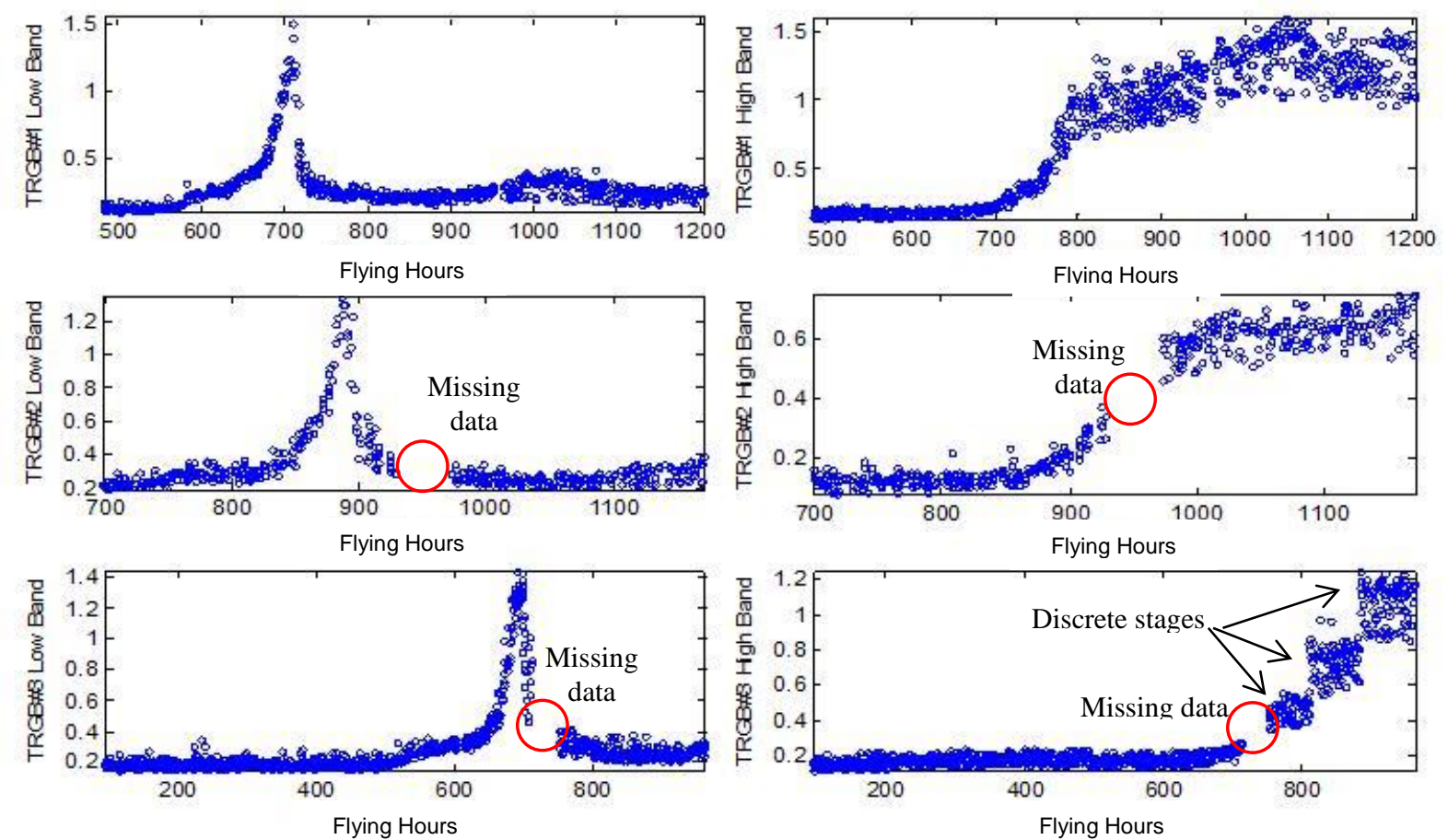

Figure 8 Low band (left) and high band feature (right) plots from defective TRGBs

It can be seen that the low band feature rises exponentially before falling back to normal levels. The exponential rise in vibration energy is commonly seen in bearing tests as shown by Harris and Kotzalas in [14]. The subsequent drop in the vibration energy is less frequently seen but tests on bearings performed by Dempsey et al [15] and Williams et al [13] had also shown similar drops. The Low band Feature is especially encouraging for further prognostic 
work as the peak feature values is consistent between the three TRGBs at $\sim 1.4 \mathrm{~g}_{\mathrm{rms}}$. However, it does not rise monotonously and falls back to normal levels as bearing damage further progresses. For the high frequency band BE feature, the vibration energy begins to rise when the low band feature reaches its peak as shown in Figure 7 with increasing scatter. The high band feature does not rise continuously but rather saturates to form an ' $S$ ' shaped profile. From the high band feature trend, it can also be seen that the Stage IV damage can progress continuously as seen in TRGB\#1 and 2 or in discrete stages can be observed for the progression in TRGB\#3.

\section{Evaluation of Bearing Time-To-Failure}

The features trends above can be used to develop prognostic models but it is used here to determine the bearing time-to-failure (TTF) first. The bearing's TTF from the detection of bearing damage is useful for initial maintenance planning, especially since prognostic algorithms tends to be inaccurate in initial estimates and improve closer to the actual failure. It should be noted that the TRGBs are in service for considerable time after the low band feature have peaked and for the ' $S$ ' shape profile for the high band feature to be formed. The grease leak is a secondary defect that occurred after the bearing has already failed. As such, the time at which the grease leak occurred does not reflect the bearing's actual time of failure. Using the feature trends, the TTF of the output shaft bearing can be estimated from the three failure cases. Failure is defined here to be the degraded state at which bearing replacement is desired. As both Stage III and IV damage can be monitored, different prognostic models can be developed depending on the damage states of interest. Figure 9 depicts the feature trends at the different stages of bearing damage.

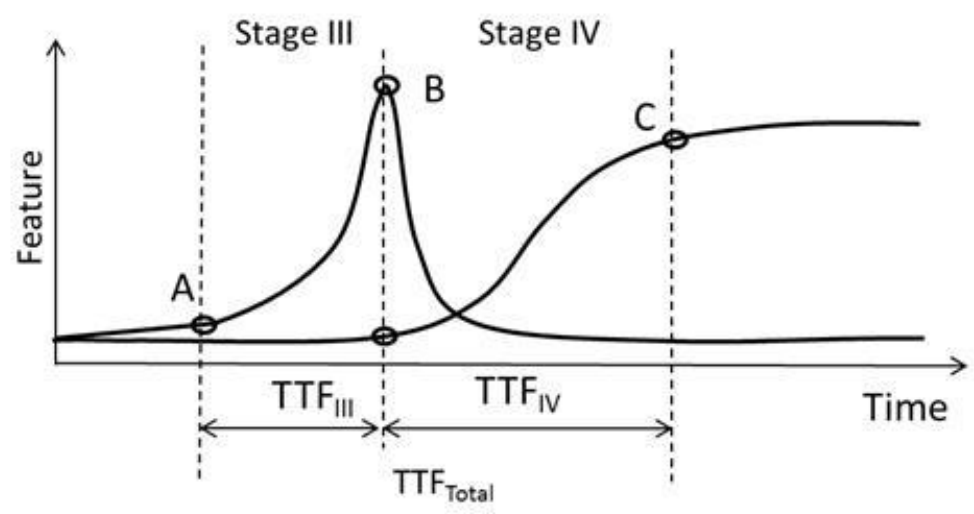

Figure 9 Features trends at different bearing damage states

If the failure condition is based on Stage III damage, the low band feature can be used with an exponential regression model to determine the time before the feature reaches an established threshold. Similarly, a regression model may be developed using the high frequency feature if the Stage IV failure condition is used. By using both models, the TTF of the bearing can be optimized as shown in Figure 9. In most run-to-failure tests, damage is initiated during the start of the test and damage propagation begins immediately. In practice, components are not seeded with defects and the time when damage initiates can vary widely and dependent on many operating and environmental factors. Therefore, the time since new is 
not evaluated and the time from detectable damage to the defined damage state is adopted instead.

\subsection{Detectable Damage Threshold}

The detectable damage threshold has to be set to reliably detect degradation in the bearing. However, there is always a trade-off between detection sensitivity and false alarm rate. A low damage threshold will be more responsive to damage but more false alarms can occur as well. In order to establish the detectable damage threshold, signal detection theory as described by Dempsey et al [8] is applied. The low and high band feature data from serviceable TRGB are obtained and their probability density distribution plots are shown in Figure 10. For both the low and high band features, the threshold is set such that the probability of false alarm from a serviceable TRGB is $\sim 0 \%$. This is well below the 5\% allowable limit as required in AC29MG15 [16] and ADS-79B [17]. As seen in Figure 9, a lognormal distribution fits both histograms of the feature data well and the threshold is set at $0.32 \mathrm{~g}(\mathrm{rms})$ and $0.27 \mathrm{~g}(\mathrm{rms})$ for the low and high band feature respectively with $\sim 0 \%$ false alarm.
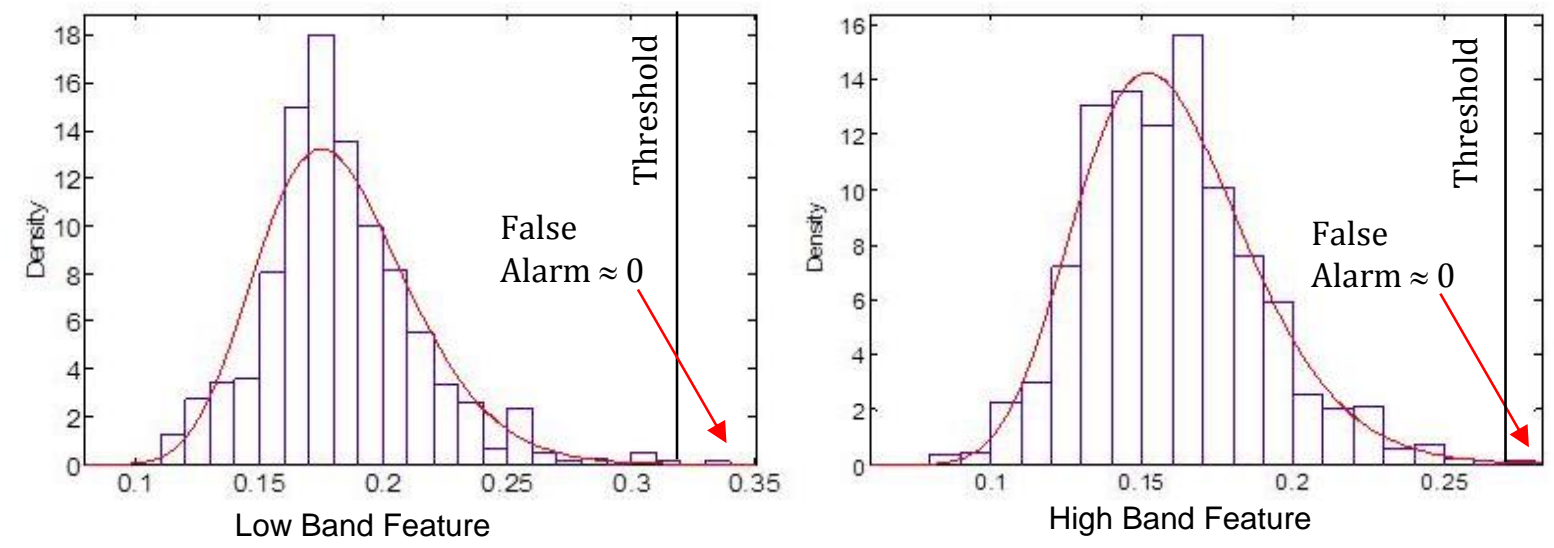

Figure 10 Detectable damage threshold setting based on low and high band feature probability density distribution from serviceable TRGBs

\subsection{Stage III Time-to-Failure}

For Stage III TTF, only the monotonic rising portion of the Low band feature is considered. The exponential function in Eqn. (1) is used to fit and estimate the regression parameters. As the trend after damage detection is of interest, the curves are aligned at the time when the detectable damage threshold is expected to be crossed. Figure 11 shows the low band feature exponential fit for the three TRGBs.

$$
f(t)=a e^{b t}+c
$$




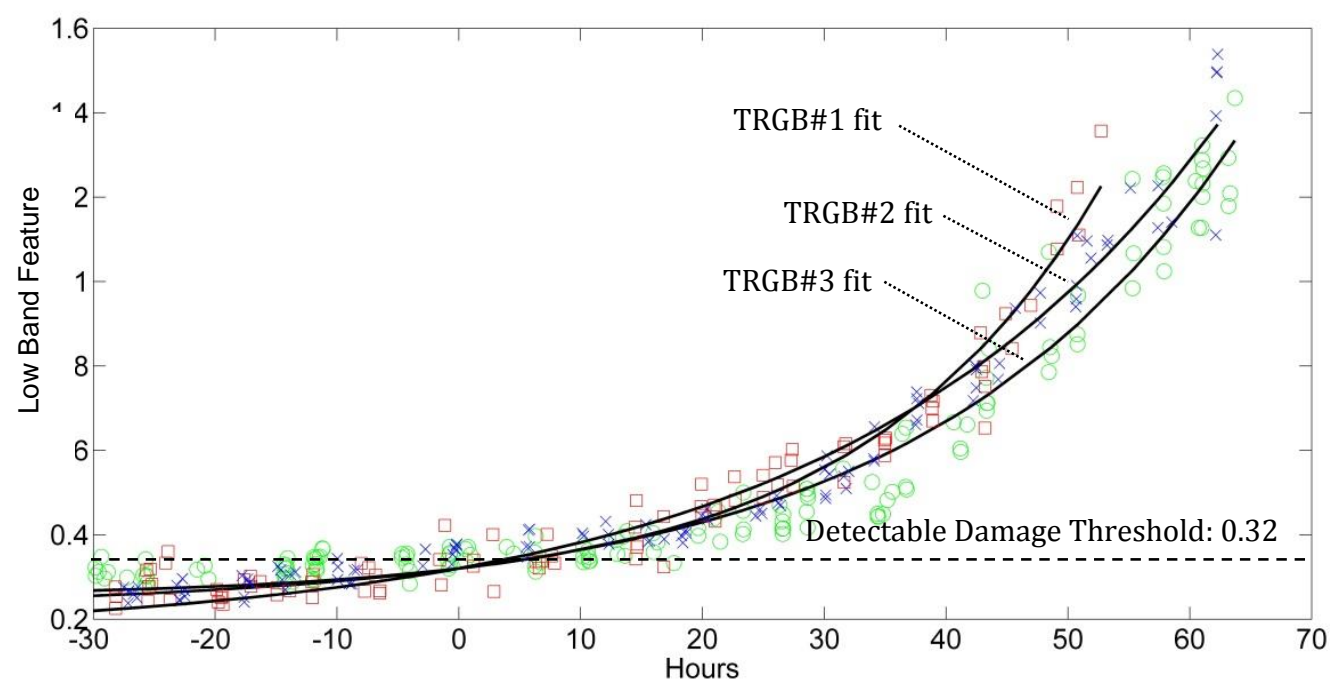

Figure 11 Exponential fit of the low band feature: ( $\square$ ) TRGB\#1, (x) TRGB\#2, (o) TRGB\#3

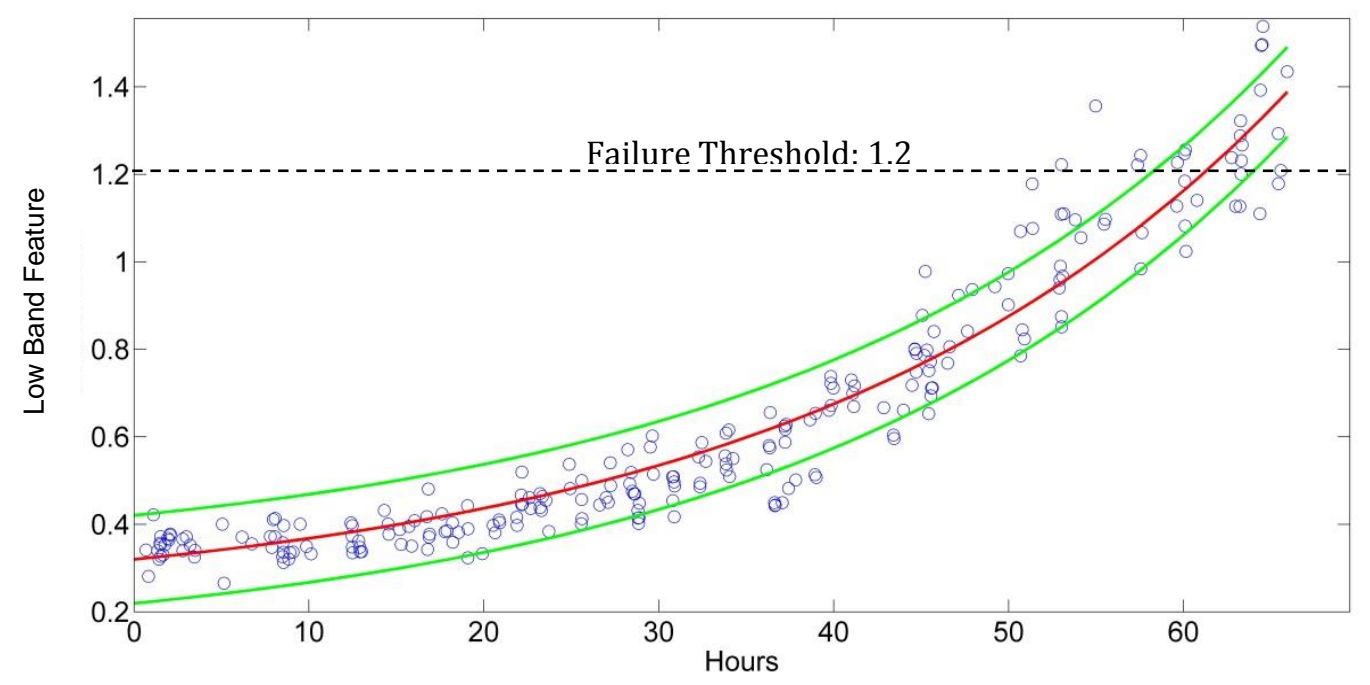

Figure 12 Exponential fit of the low band feature datasets with $90 \%$ confidence bounds

It can be seen from Figure 11 that the three plots correlate very closely which shows that the rate of damage progression for Stage III damage is consistent. A regression curve is fitted to the combined dataset to estimate the overall degradation path in Figure 12. The peak values for the low band features are similar between the three gearboxes at $1.54 \mathrm{~g}_{\mathrm{rms}}, 1.36 \mathrm{~g}_{\mathrm{rms}}$ and $1.44 \mathrm{~g}_{\mathrm{rms}}$ respectively. As such, the failure threshold for Stage III damage is conservatively set lower at $1.2 \mathrm{~g}_{\mathrm{rms}}$. From the regression fit in Figure 12, the time from detectable damage of $0.32 \mathrm{~g}_{\mathrm{rms}}$ to the defined localised damage threshold of $1.2 \mathrm{~g}_{\mathrm{rms}}$ is determined to be $61.1 \mathrm{Hrs}$ with corresponding $90 \%$ confidence bounds of 58.1.Hrs and 63.8Hrs. The 90\% confidence bounds were included to provide a probabilistic measure of the TTF. 


\subsection{Stage IV Time-to-Failure}

In Figure 8, the high band feature displays a ' $\mathrm{S}$ ' shaped profile also known as a logistic function with increased scatter. As such, a 5-parameter logistic (5PL) regression model as shown in Eqn (2) is used for the fitting the high band features. The 5PL model is used as it can flexibly fit asymmetric trends in the data compared to standard logistic regression as described in [18]. However, it is noted that the 5PL curve can be difficult to fit as the initial estimate of each parameter has to be selected carefully. In this study, the initial estimates are selected through trial and error and adjusting the parameters based on their properties shown in Table 2.

$$
f(t)=a+\frac{b}{\left(1+\left(\frac{t}{c}\right)^{d}\right)^{e}}
$$

Table 2 Properties of the 5PL parameters

\begin{tabular}{ll}
\hline Parameter & Properties of curve \\
\hline A & Lower asymptote \\
B & Upper asymptote \\
C & Affects the position of inflection point \\
D & Rate of change between asymptotes \\
E & Asymmetry factor \\
\hline
\end{tabular}

The fitted curves for the three gearboxes are shown in Figure 13 and the fitted parameters. The curves are aligned at the time when the low band feature reaches their peak values. Unlike the low band feature, the high band curves do not have similar limits. The upper asymptotes for the three TRGBs from the fitted curves are $1.13 \mathrm{~g}_{\mathrm{rms}}, 0.65 \mathrm{~g}_{\mathrm{rms}}$ and $1.17 \mathrm{~g}_{\mathrm{rms}}$ respectively. The logistic regression is performed on the combined high band feature dataset as shown in Figure 14. Due to the increasing variance (or heteroscedasticity) in the data, a robust regression; where outlier has decreasing weight in the regression, is employed. For a conservative estimate of the generalised damage failure time, a low failure threshold of 0.5 $\mathrm{g}_{\mathrm{rms}}$; which is below the upper asymptote of TRGB\#2 is set. Based on this threshold, the Stage IV TTF is $72.4 \mathrm{Hrs}$ corresponding $90 \%$ confidence band of $14.3 \mathrm{Hrs}$ and $133.6 \mathrm{Hrs}$. The Stage IV TTF is less accurate compared to the Stage III TTF as the confidence bounds are much wider. For higher confidence levels above 90\%, the Stage IV model would be ineffective as the confidence bounds would be too wide for any effective TTF to be estimated. 


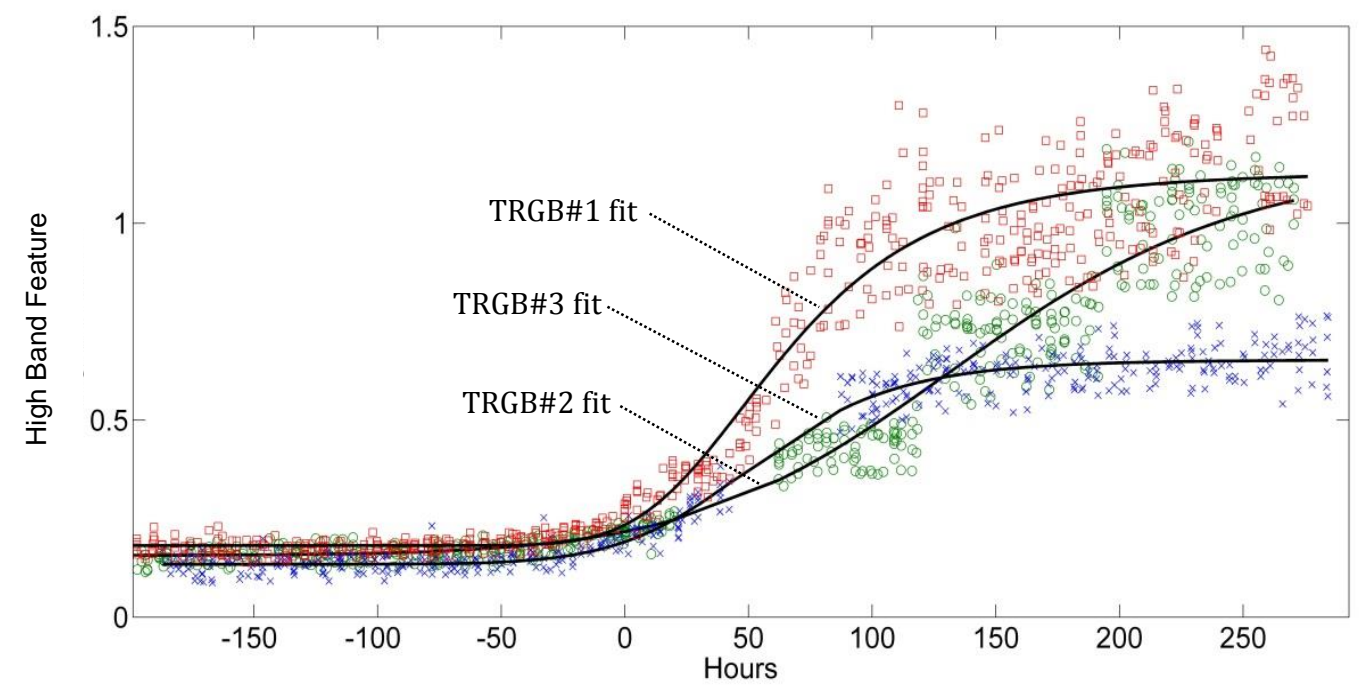

Figure 13 5PL fit of the High Band Feature: ( $\square$ ) TRGB\#1, (x) TRGB\#2, (o) TRGB\#3

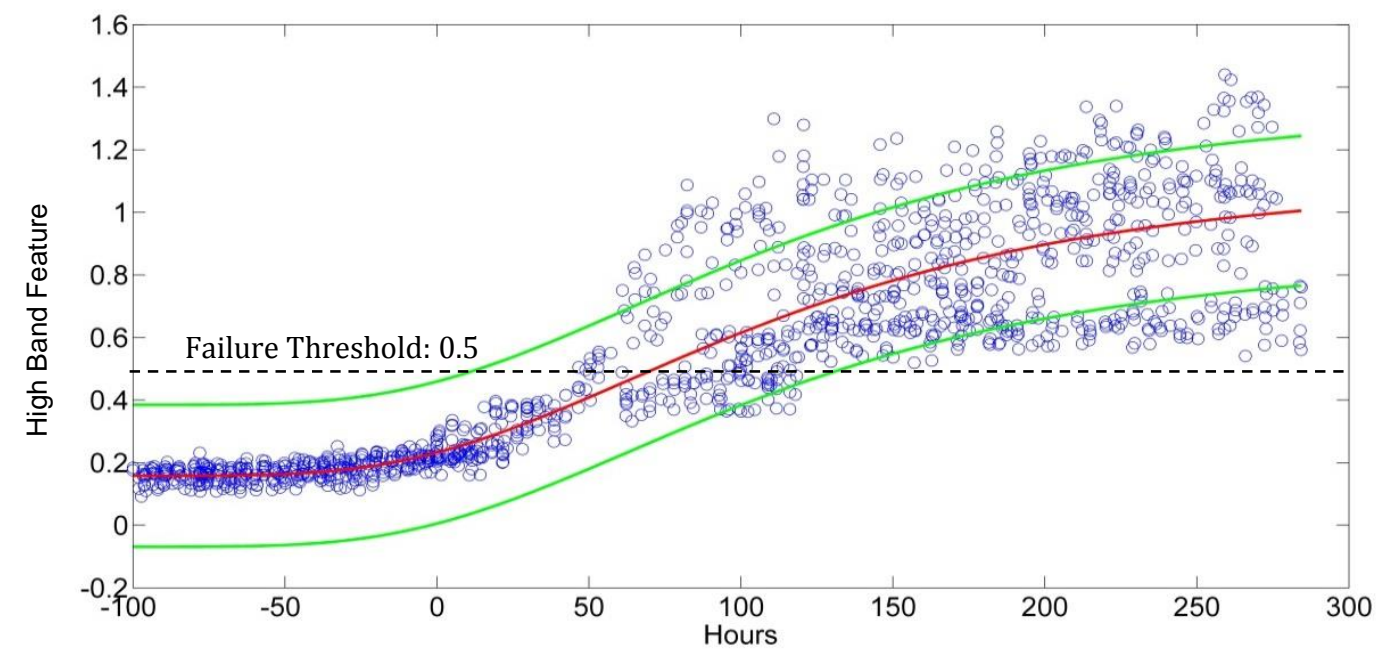

Figure 14 5PL fit of the High Band Feature with 90\% confidence bounds

\subsection{Bearing Time-To-Failure}

In most bearing analysis literature, the focus is mainly on diagnosing incipient bearing defects and using it for remaining useful life prediction. In practice, most bearing is still functional till the damage is widespread throughout as most bearings are designed and used based on safe-life philosophy. As such, TTF based on Stage III damage only does not optimize the economic service life of the bearing. In this application, use of both Stage III and IV damage is preferred as it is already shown that the TRGB can operate with Stage IV damage for a considerable time. Nonetheless, the TTF from damage detection to widespread damage is still required for maintenance planning purpose. From analysis of the feature trends, the TTF for both Stage III and Stage IV bearing damage were estimated. Assuming a 
normal distribution, $\mathrm{TTF}_{\text {III }}$ is $\sim N\left(61.6,2.1^{2}\right)$ and $\mathrm{TTF}_{\mathrm{IV}}$ is $\sim N\left(72.4,47.7^{2}\right)$. The sum of both failure times can therefore be evaluated to be $\mathrm{TTF}_{\text {total }} \sim N\left(134,47.8^{2}\right)$. Using both Stage III and IV damage model, the $90 \%$ lower confidence of the $\mathrm{TTF}_{\text {total }}$ is $72.7 \mathrm{Hrs}$ and the upper bound is $195.3 \mathrm{Hrs}$. Using only the lower confidence bound for conservative estimate, the combined models allows a $25 \%$ increase in the detection lead-time compared to the use of Stage III damage model alone.

\section{Maintenance Application}

From these findings, a CBM program for the TRGB output shaft bearing can be recommended. The bearing can be monitored for Stage III damage using the detectable damage threshold established in Section 7.1. In event that this threshold is exceeded, the replacement of the TRGB can be planned for in the next 72.7 Hrs. For more accurate assessment of the remaining useful life of the bearing after detection, regression-based prognostics model; such as one performed by Siegel et al [19], can be performed. The use of low band features is particularly well-suited as its trend and threshold limit are fairly consistent compared to High Band Features. The use of prognostic model however is not explored further here. The high band feature can also serve as a diagnostic tool as the rise in magnitude and increased scatter of the feature are clear indication of widespread damage within the bearing. This redundancy can be useful for this TRGB application as the low band feature does not rise continuously and will drop back to normal levels after it peaks. If the HUMS data during the period in which the low band feature is unavailable, the high band feature can still provide indication of bearing damage at a later time. The low band feature trends for

\section{Conclusion}

In this study, the operational HUMS data from three TRGBs found with damaged bearings were analyzed and correlated with their tear-down inspection findings. From analysis of their vibration spectrum, it was shown that there were fault patterns that distinguish the TRGBs with damaged bearings from serviceable ones clearly and agrees very well with established bearing damage models. From these fault patterns in the vibration spectrum, the progression from localised damage in Stage III and subsequently widespread damage in Stage IV can be inferred. Two features were then developed from selected frequency bands in the vibration spectrum to monitor the bearing damage stages. Besides demonstrating that spectral analysis can be effective in the field environment, considerations in the use of HUMS data for maintenance applications are presented. Using both low and high and features, the lead time between damage detection and bearing replacement is improved.

\section{References:}

[1] Jardine, A. K. S., Lin, D. and Banjevic, D. (2006), "A review on machinery diagnostics and prognostics implementing condition-based maintenance", Mechanical Systems and Signal Processing, vol. 20, no. 7, pp. 1483-1510. 
[2] Heng, A., Zhang, S., Tan, A. C. C. and Mathew, J. (2009), "Rotating machinery prognostics: State of the art, challenges and opportunities", Mechanical Systems and Signal Processing, vol. 23, no. 3, pp. 724-739.

[3] Baillie, D. C. and Mathew, J. (1996), "A comparison of autoregressive modeling techqniues for fault diagnosis of rolling element bearings", Mechanical Systems and Signal Processing, vol. 10, no. 1, pp. 1-17.

[4] Uluyol, O., Kim, K. and Hickenbottom, C. (2011), "Update to a Systematic Approach to Bearing Health Monitoring", 7th DSTO International Conference on Health \& Usage Monitoring, 28th Feb - 3rd Mar, Melbourne, Australia, .

[5] Dykas, B., Krantz, T.,L., Decker, H. and Lewicki, D.,G (2009), Experimental Determination of AH64 Apache Tailshaft Hanger Bearing Vibration Characteristics with Seeded Faults, ARL-TR-4865, Army Research Laboratory, USA.

[6] Randall, R. B. (2011), Vibration-based condition monitoring : industrial, aerospace, and automotive applications, Wiley, Chichester.

[7] Sikorska, J. Z., Hodkiewicz, M. and Ma, L. (2011), "Prognostic modelling options for remaining useful life estimation by industry", Mechanical Systems and Signal Processing, vol. 25, no. 5, pp. 1803-1836.

[8] BS ISO 15243:2004 (2004), "<br />Rolling bearings - Damage and failures - Terms, characteristics and causes", British Standards Institution, .

[9] Nicholas, G., Abdel, B., Vytautas, B., Ronak, S. and Yong-June, S. (2009), "CBM Component Testing at the University of South Carolina: AH64 Tail Rotor Gearbox Studies", American Helicopter Society Technical Specialists' Meeting on Condition Based Maintenance, Feb 10-11, Huntsville, AL, USA, .

[10] STI Field Application Note (2012), Rolling Element Bearings, REB, Sales Technology, Inc., League City TX.

[11] Qiu, H., Luo, H. and Eklund, N. (2009), On-board bearing prognostics in aircraft engine: Enveloping analysis or FFT? AFRL-RX- WP - TP -2009-4141, Air Force Research Laboratory, USA.

[12] M., E. and D., M. "Assessment of natural crack initiation and its propagation in slow speed bearings", Nondestructive Testing and Evaluation, vol. 24, no. 3, pp. 261-275.

[13] Williams, T., Ribadeneira, X., Billington, S. and Kurfess, T. (2001), "Rolling Element Bearing Diagnostics in Run-to-Failure Lifetime Testing", Mechanical Systems and Signal Processing, vol. 15, no. 5, pp. 979-993.

[14] Kotzalas, M. N. and Harris, T. A. (2001), "Fatigue Failure Progression in Ball Bearings", Journal of Tribology, vol. 123, no. 2, pp. 238-242. 
[15] Paula, J., Dempsey, Nathan, B., Chris, H., Jeremy, B. and Daniel, R., Wade (2010), "Correlate Life Predictions and Condition Indicators in Helicopter Tail Gearbox Bearings", American Helicopter Society 66th Annual Forum, May 11-13, Phoenix, AZ, USA, .

[16] Federal Aviation Adminstration, ( 2003), AC29 MG15 - Airworthiness Approval of Rotorcraft Health Usage Monitoring Systems (HUMS), USA.

[17] Army Aviation and Missile Life Cycle Command, ( 2011), ADS-79B-HDBK (2011), Handbook for Condition Based Maintenance Systems for US Army aircrafts, USA.

[18] Gottschalk, P. G. and Dunn, J. R. (2005), "The five-parameter logistic: A characterization and comparison with the four-parameter logistic", Analytical Biochemistry, vol. 343 , no. 1 , pp. 54-65.

[19] Siegel, D., Ly, C. and Lee, J. (2012), "Methodology and Framework for Predicting Helicopter Rolling Element Bearing Failure", Reliability, IEEE Transactions on, vol. 61, no. 4, pp. 846-857. 